\title{
Wortstellungsvariation und Konstruktionsverschmelzung
}

\begin{abstract}
Die Möglichkeit, Präpositionalphrasen aus Nominalphrasen zu extrahieren, unterliegt bestimmten Einschränkungen, vgl.: Über dieses Thema schreibt sie ihre Hausarbeit, Über den Zweiten Weltkrieg hat er viele Bücher gelesen, aber: *Über Magie haben sie alle Bücher vernichtet (Alle Bücher über Magie haben sie vernichtet), ?? ̈̈ber die Liebe möchte er ein Buch bestellen (Ein Buch über die Liebe möchte er bestellen). Im Beitrag wird argumentiert, dass die Extraktion von Präpositionalphrasen mit konstruktionellen Faktoren in Zusammenhang gebracht werden kann, die über pragmatische Motive hinausgehen. Sätze, in denen zwei sich überlappende Konstruktionen instanziiert werden, weisen eine größere Wortstellungsvariation auf als Sätze mit nur einer der beiden Konstruktionen.

There are certain constraints on extracting prepositional phrases from noun phrases, in German as well as in many other languages; compare: Über dieses Thema schreibt sie ihre Hausarbeit, Über den Zweiten Weltkrieg hat er viele Bücher gelesen, but: *Über Magie haben sie alle Bücher vernichtet (Alle Bücher über Magie haben sie vernichtet), ?"Über die Liebe möchte er ein Buch bestellen (Ein Buch über die Liebe möchte er bestellen). The extraction of prepositional phrases can be accommodated in a constructional account that goes beyond an explanation on pragmatic grounds. Clauses that instantiate two partly overlapping constructional templates - a kind of "pattern imbrication" - exhibit more word order variation than clauses realizing only one of the templates.
\end{abstract}

\section{Einführung}

Inseln, Barrieren, Extraktionen, Extrapositionen und ähnliche Phänomene spielen im Umfeld der generativen Grammatik eine wichtige Rolle (vgl. u.a. Chomsky 1986, 2005; Pafel 1995; Müller 2003; Broekhuis 2005). Durch solche Analysen wurde das Augenmerk auf Phänomene gerichtet, die bei weniger formal orientierten grammatischen Analysen bisher weniger im Fokus standen, bei denen aber eine funktionale Deutung zusätzliche Erkenntnisse verspricht. Das soll im Folgenden am Beispiel der Extraktion gezeigt werden.

Warum ist z.B. Satz (1b) syntaktisch unproblematisch (wenn auch markiert), nicht aber Satz (2b) (Beispiele aus Erteschik-Shir 1981, S. 665)?

(1a) John wrote a book about Nixon.

(1b) Who did John write a book about?

(2a) John destroyed a book about Nixon.

(2b) *Who did John destroy a book about?

In der modernen Linguistik wird die Versetzung des Frageworts who an den Anfang des Satzes in (1b) als ein Beispiel von Extraktion aus der Nominalphrase (NP) a book about Nixon bezeichnet, die in (1a) als direktes Objekt (DO) fungiert (s. Pafel 1995, S. 145f.). Zur Erklärung des Unterschieds zwischen (1b) und (2b) greifen Autoren wie ErteschikShir (1981) und Bouchard (1995, 2013) auf das Konzept der „Dominanz“"zurück, das kein formallinguistisches, sondern ein pragmatisches Konzept darstellt. Es besagt, dass ein Sprachelement „dominant“ ist, wenn ein Sprecher die Aufmerksamkeit des Hörers auf die Bedeutung eben dieses Elementes und kein anderes lenken möchte (,the speaker intends 
to direct the hearer's attention to the intension of c [constituent]“, Erteschik-Shir 1981, S. 665). In (1a) werde hervorgehoben, dass John nicht irgendein, sondern ein Buch über Nixon schreibe, während in (2a) ausgedrückt werde, dass John ein Buch vernichte, eins, das darüber hinaus von Nixon handle. In (1a) liege der Fokus auf dem Inhalt des Buches about Nixon, in (2a) auf dem Inhalt des Verbs destroy, vernichten, zerstören'. Nur was in der Präpositionalphrase (PP) „,dominant“ sei, könne extrahiert werden, und das erkläre, weshalb (1b) einwandfrei sei, nicht aber (2b) (Erteschik-Shir 1981, S. 667). Übrigens lassen sich - wie bei fast allen pragmatischen Motivationen im Sprachgebrauch - Kontexte erfinden, in denen auch ein Satz wie (2b) akzeptabler wird, z.B. wenn jedermann weiß, dass John ein professioneller Büchervernichter ist (Erteschik-Shir 1981, S. 667 und Bouchard 1995, S. 367-375; 2013, S. 309). Was „Dominanz“ heißt, erweist sich somit auch als kontextsensitiv.

Es gibt weitere Faktoren, die bei der Erklärung der Extraktion herangezogen werden. So könne eine PP, die semantisch eng mit der NP zusammenhängt, leichter extrahiert werden als eine PP, die semantisch nur lose mit der NP verbunden ist. Aufgrund dieser Unterscheidung wird die PP dann entweder als Ergänzung der NP (,complement PP“) oder als Angabe (,,adjunct PP“) bezeichnet. Van de Velde (2012, S. 448-449) gibt für diese Unterscheidung u.a. folgende Beispiele aus dem Englischen: the need for donors, the father of the boy, the care for his brother (,complement PPS"), a man from China, the train in the afternoon, the man without a face (,,adjunct PPs"). Belege aus dem Deutschen Referenzkorpus (DeREKo; www.ids-mannheim.de/kl/projekte/korpora/) zeigen indes, dass das Kriterium des semantischen Zusammenhangs und die Unterscheidung der beiden Typen von PP für eine Erklärung von Extraktion im Deutschen wenig tauglich sind. Gemäß dem Wörterbuch werden z.B. präpositionale Ergänzungen beim Nomen Experte mit den Präpositionen für oder in gebildet (vgl. z.B. Duden 1999, S. 1137). Eine entsprechende Variation findet sich auch in den Korpusbelegen:

(3) Der Landkreis sollte vielmehr einen Experten für Förderanträge einstellen. (Braunschweiger Zeitung, 24.8.2011)

(4) Mit dem Vizepräsidenten des Landesverbandes, Axel Rott, hatten sie zugleich einen Experten in Waffenrecht zu Gast. (Braunschweiger Zeitung, 16.3.2009)

Es sind jedoch nur Sätze mit linksversetzter für-PP wie (5) belegt:

(5) Für jede Waffengattung gibt es Experten. (die tageszeitung, 25.2.2003)

Dagegen erscheint eine Äußerung wie (6) im analogen Kontext nicht normgemäß:

(6) ??In Waffenrecht hatten sie einen Experten zu Gast.

Auf der anderen Seite geht aus den Belegen (7)-(8) hervor, dass die von-PP bei einem Nomen wie Exemplar sowohl direkt neben der NP als auch am Satzanfang stehen kann, womit aber offensichtlich nichts über ihren Status als Ergänzung oder Angabe gesagt ist:

(7) Die Besonderheit ihrer Schmuckkreationen ist, dass sie nur drei Exemplare von jedem Stück herstellt. (Niederösterreichische Nachrichten, 6.3.2007, S. 43)

(8) Von Antons Hocker wird die Firma „Löffler Bürositzmöbel“ fünf Exemplare herstellen. (Nürnberger Zeitung, 27.7.2011, S. 2)

Schließlich ist die über-PP in Beleg (9a) keine Ergänzung zur NP einen Stern, die Linksversetzung der PP (9b) bereitet aber keine Probleme: 
(9a) Hirten wanderten durch die Gegend und beobachteten einen Stern über Bethlehem, der immer heller wurde. (St. Galler Tagblatt, 22.12.1999)

(9b) Über Bethlehem beobachteten Hirten einen Stern, der immer heller wurde.

Aus Beobachtungen wie den obigen geht hervor, dass PPs, die in sehr unterschiedlichem Maße an NPs gebunden sind, an den Satzanfang rücken können. Weil die Wortstellungsvariation von PPs darüber hinaus mit mehreren und verschiedenartigen Faktoren zusammenhängt, die womöglich bis auf die Ebene von Einzellexemen (Verb, Nomen, Präposition, Artikel) und deren Valenz und Semantik hinabreichen, fasst man sie am besten als ein Kontinuum auf. Eine allesumfassende Darstellung, die alle Aspekte der Wortstellungsvariation auf einmal erklären will, erscheint nicht realistisch. Es dürfte ergiebiger sein, spezifische Strukturen in Augenschein zu nehmen und auf mögliche semantische und syntaktische Gründe hin zu analysieren. In diesem Beitrag werden Beispiele aus der deutschen Gegenwartssprache untersucht, bei denen Extraktion einer PP aus einer NP, die in einem Aktivsatz als DO fungiert, vorliegt. ${ }^{1}$ Dabei wird es erforderlich sein, den Begriff der Extraktion selbst noch genauer zu bestimmen, weil ja nicht jede Möglichkeit, eine PP statt direkt neben einer NP an den Satzanfang zu stellen, eine Extraktion darstellt (vgl. z.B. die Sätze (3)-(5) und (7)-(8)). Darüber hinaus darf der Terminus „Extraktion“, sofern man damit ein „dynamisches“ Verfahren der Herausstellung bezeichnet, nicht darüber hinwegtäuschen, dass man in der Sprache auch ein syntaktisches Muster mit vorangestellter PP annehmen kann, das eine eigenständige Konstruktion der Sprache darstellt. ${ }^{2}$

\section{Wortstellungsvariation bei PPs mit der Präposition über}

Schauen wir uns die beiden folgenden Reihen von (frei erfundenen) Sätzen mit über an, die den obigen englischen Beispielsätzen (1)-(2) nachempfunden sind: ${ }^{3}$

(10a) Paul schreibt eine Hausarbeit über Willy Brandt.

(10b) Eine Hausarbeit über Willy Brandt muss er schreiben.

(10c) Über Willy Brandt muss er eine Hausarbeit schreiben.

(10d) Über wen schreibt Paul seine Hausarbeit?

(10e) Über Willy Brandt muss er schreiben.

(10f) Paul schreibt.

(10g) Was schreibt Paul?

(11a) Doris bestellt ein Buch über Helmut Schmidt.

Für andere Typen von Extaktion s. Pafel (1995); eine Übersicht unterschiedlicher „Herausstellungen“ im Deutschen liefert Altmann (1981). Versetzung eines Sprachelements an das Ende des Satzes heißt „Extraposition“, wie z.B. in Ich habe ein Buch mit rotem Einband gekauft > Ich habe ein Buch gekauft mit rotem Einband (die Extraposition ist grammatisch), dagegen *Mit rotem Einband habe ich ein Buch gekauft (keine Extraktion möglich) (Pafel 1995, S. 151-152).

2 Dementsprechend spreche ich, wie in der einschlägigen Literatur üblich, auch dann von „Extraktion“, wenn genau genommen die „Möglichkeit zur Extraktion“ als Konstruktion gemeint ist. Die im Folgenden entwickelte Erklärung dürfte über weite Strecken auch für PPs aufgehen, die nicht das DO des Satzes bilden (vgl. Eine Dissertatioon über Willy Brandt wäre längst fällig > Über Willy Brand wäre eine Dissertation längst fällig), der Nachweis dafür erfordert aber eine gesonderte Analyse.

3 Im Gegensatz zum Englischen gibt es in der deutschen Standardsprache kein sog. Stranden der Präposition (wie im englischen Beispiel (1b)), was uns hier aber nicht weiter beschäftigt. Stranden ist übrigens in bestimmten deutschen Dialekten belegt (Pafel 1995, S. 164). 
(11b) Ein Buch über Helmut Schmidt möchte sie bestellen.

(11c) ?"Über Helmut Schmidt möchte Doris ein Buch bestellen. ${ }^{4}$

(11d) ?? Über wen bestellt Doris ein Buch?

(11e) ??Über Helmut Schmidt möchte sie bestellen.

(11f) ??Doris bestellt.

(11g) Was bestellt Doris?

Es stellt sich die Frage, worauf die Einschränkungen der Wortstellungsvariation unter (11) zurückzuführen sind. Sicherlich kann man die unterschiedlichen Extraktionsmöglichkeiten der PP in (10) und (11) mit dem oben erwähnten Prinzip der Dominanz in der Informationsübertragung in Verbindung bringen. Immerhin betrifft das Bestellen eines Buches in (11) (wie auch dessen Vernichtung im englischen Beispielsatz (2)) den materiellen Gegenstand und erst in zweiter Linie dessen Inhalt - obwohl der Inhalt in der Regel natürlich der Grund dafür ist, dass das materielle Buch bestellt wird. ${ }^{5}$ Dagegen schreibt man eine Hausarbeit über ein bestimmtes Thema, so dass der Inhalt der Arbeit der eigentliche Zweck des Schreibens ist und nicht ihre materielle Beschaffenheit, die in der Regel zweitrangig sein dürfte. Das Dominanzprinzip ist darüber hinaus geeignet, die unterschiedlichen Satzbedeutungen von (10b) Eine Hausarbeit über Willy Brandt muss er schreiben und (10c) Über Willy Brandt muss er eine Hausarbeit schreiben zu erhellen. Die Voranstellung der PP in (10c) gestattet offensichtlich einen besonderen Fokus, der informationsstrukturell durch die Voranstellung der gesamten Objekt-PP-Fügung in (10b) nicht möglich erscheint. Zwei Korpusbelege können das weiter veranschaulichen:

(12) Eine Lehrerin im Kibbuz meint zwar: „Über das Ghetto kann man keinen Roman schreiben." (Nürnberger Nachrichten, 30.3.2006)

(13) Über jeden Ausflug müssen die Kinder einen Bericht schreiben. (Nürnberger Zeitung, 12.8.2008, S. 2)

Man beachte, dass die satzsemantische Leistung der Herausstellung der PP an den Satzanfang nur vor dem Hintergrund der Wortstellungsvariation im weiteren Sinne bestimmt werden kann, vgl.: Man kann über das Ghetto keinen Roman schreiben im Gegensatz zu Einen Roman über das Ghetto kann man nicht schreiben (grammatisch, aber mit anderer Satzbedeutung), dagegen auch: Man kann keinen Roman über das Ghetto schreiben (ohne speziellen Fokus auf die PP). In diesem Beitrag werde ich mich weiterhin auf Herausstellung an den Satzanfang konzentrieren.

Auf die Diskussion von Kontexten, in denen die unter (11) mit zwei Fragezeichen versehenen Beispiele als akzeptabler erscheinen könnten, wird in diesem Beitrag verzichtet. Generell ist dieser Punkt zweifellos bedeutsam, und das Phänomen, dass auf den ersten Blick inakzeptable Sätze (oder sogar vermeintlich „ungrammatische“ Sätze, vgl. (2b)) bei geeigneter Kontextualisierung viel von ihrer scheinbaren Inakzeptabilität verlieren, sollte theoretisch wie methodologisch angemessen berücksichtigt werden. Vielfach erscheinen solche Sätze nur in Isolation nicht annehmbar. Die sprachliche Kreativität von Sprechern

$4 \quad$ Interpretiert man (11c) so, dass Helmut Schmidt eine Mittelperson bezeichnet, dann ist der Satz ohne Weiteres grammatisch. Weil in dem Fall aber keine Extraktion vorliegt, braucht uns diese Interpretation hier nicht weiter zu beschäftigen (vgl. auch (20)).

5 Weil die beiden Interpretationen eines Wortes wie Buch relativ deutlich abgrenzbar sind und mit typischen sprachlichen Kontexten korrelieren, lässt sich diese Form von Alternanz unter „,komplementäre Polysemie“ einreihen (vgl. Pustejovsky 1995, S. 31, 77ff. und 101). 
und Hörern sorgt oft für pragmatische, semantische und/oder intonatorische Anpassungen und Berichtigungen, mittels derer die Striktheit der Grenze zwischen akzeptabel und inakzeptabel deutlich relativiert wird (vgl. auch Wasow/Arnold 2005 und Dabrowska 2010). So kann man sicherlich einen Kontext entwerfen, in dem ein Satz wie (11c) ?? ̈̈ber Helmut Schmidt möchte Doris ein Buch bestellen annehmbar erscheint, z.B. wenn man einen expliziten Kontrast herstellt, wenn zuvor etwa von einem Buch über Willy Brandt die Rede war. Dennoch ist der Gebrauch von Fragezeichen wie in (11) berechtigt, wenn damit auf die Feststellung Bezug genommen wird, dass bestimmte Ausdrücke in Korpora nicht vorkommen. Unter diesem Aspekt gilt es in diesem Beitrag herauszufinden, weshalb außer Sätzen wie X schreibt ein Buch über $Y$, X bestellt ein Buch über $Y$ und Xvernichtet Bücher über $Y$ zwar der Satz Über Y schreibt X ein Buch belegt werden kann, nicht aber Über $Y$ bestellt X ein Buch und Über Y vernichtet X Bücher, auch wenn die letzteren Sätze unter bestimmten Umständen (z.B. Kontrast) akzeptabel sein können. ${ }^{6}$

Wenn man dann auf das Konzept der „Dominanz“ zurückgreift, muss man bedenken, dass die Hervorhebungen, die es erfassen soll, grundsätzlich für alle inhaltstragenden Konstituenten in einem Satz möglich sind. Demnach kann dieses Konzept zwar die zunehmende Akzeptabilität von ursprünglich mit Fragezeichen oder Sternen versehenen Sätzen begründen helfen, es reicht aber nicht aus, zu erklären, warum z.B. Über Y bestellt X ein Buch und Über $Y$ vernichtet $X$ Bücher zunächst als weniger akzeptabel eingestuft werden müssen, während der entsprechende Satz Über $Y$ schreibt $X$ ein Buch von vornherein als unproblematisch gilt. Es empfiehlt sich deshalb, weitere Gründe für die Wortstellungsvariation ausfindig zu machen. Im nächsten Abschnitt wird für diese Verhältnisse eine konstruktionelle Erklärung vorgeschlagen.

\section{Konstruktionsverschmelzung}

Man kann die unter (10) aufgeführte Wortstellungsvariation, die unter (11) keine unmittelbare Entsprechung findet, außer mit inhaltlichen Hervorhebungen mit den Konstruktionen in Zusammenhang bringen, die im Satz instanziiert werden und die als solche über einen pragmatischen Faktor wie die besprochene Dominanz hinausgehen. Unter „Konstruktion“ verstehe ich ganz allgemein ein komplexes Form-Bedeutungsgebilde, so wie man es in heutigen konstruktionistischen Ansätzen definiert. ${ }^{7}$ Von den verschiedenen Konstruktionen, die hier angenommen werden, sind die sog. Argumentstrukturkonstruktionen am besten erforscht. Wenn man eine solche Beschreibung auf unsere Beispiele anwendet, so kann man die Argumentstrukturkonstruktion in (10a) und (11a), ohne in technische Details einzutreten, als ein Satzmuster mit transitiver Bedeutung beschreiben, das der typischen Form (14) entspricht:

[ [NPNominativ] [Vtransitiv] [NPaKkUSATiv] ]

\footnotetext{
Vgl. ähnlich Welke (1988, S. 26, 58ff.) über die kontrastive Weglassbarkeit valenzgebundener Ergänzungen vor dem Hintergrund einer sog. „Grundvalenz“ von Verben. Unter einer solchen Bedingung dürfte z.B. auch (11f) annehmbar sein, vgl. Man bestellt nicht, sondern es wird serviert (Blogbeleg). Beweist ein solcher Beleg, dass bestellen auch monovalent grammatisch ist, oder dass der monovalente Gebrauch von bestellen zwar ,ungrammatisch“, aber in einem besonderen Kontext dennoch akzeptabel ist? (Vgl. Coseriu 1985, S. xxxv über die pragmatisch bedingte Akzeptabilität ungrammatischer Ausdrücke.)

7 S. etwa Ziem/Lasch (2013, S. 10ff.), jedoch ohne Festlegung auf die „holistische“ kognitive Interpretation des Begriffs „Konstruktion“, die m.E. zu Unrecht sprachliche Bedeutungen und enzyklopädische sowie pragmatische Inhalte miteinander identifiziert, s. Coene/Willems (2006), Willems/Coene (2006) und Willems (2011) für kritische Auseinandersetzungen mit diesem Aspekt moderner Konstruktionsgrammatiken.
} 
Die verbale Leerstelle in diesem Muster kann in Verbindung mit geeigneten NPs durch Verben wie schreiben und bestellen instanziiert werden. (10e) und (11e) beweisen aber, dass schreiben im Gegensatz zu bestellen auch die intransitive Argumentstrukturkonstruktion mit entsprechender Bedeutung instanziieren kann, deren Muster die typische Form (15) aufweist:

\section{(15) [ [NPnominativ] [Vintransitiv] ]}

Sätze stellen gemäß dem konstruktionellen Ansatz in der Regel Kombinationen von Konstruktionen dar. In einem Satz wie (10a) z.B. kann man außer (14) auch das verbale Flexionsmuster (schreib-t), das Muster eines Determinativkompositums (Haus-arbeit), das Muster der nominalen Determinierung (eine Hausarbeit) usw. unterscheiden (zur Kombination von verschiedenen Konstruktionen in einem Satz s. Goldberg 2003, S. 221).

Von der gewöhnlichen Kombination verschiedener Konstruktionen in einem Satz unterscheidet sich das Phänomen der Konstruktionsverschmelzung in einigen Hinsichten. Wichtig ist, dass in einem konkreten Satz wie (10a) - wie auch im englischen Beispiel (1a) - zwei Konstruktionen instanziiert werden, die miteinander überlappen, indem sie sich die Leerstelle für die PP teilen. Das Ergebnis ist, dass in (10a) eine Verschmelzung von zwei teilweise ähnlichen und deshalb leicht miteinander fusionierbaren Konstruktionen vorliegt, was insofern einen Einfluss auf die Wortstellungsvariation hat, als außer der Voranstellung der gesamten Objekt-PP-Fügung auch die separate Extraktion der PP möglich ist. Dagegen liegt in (11a) - wie auch in (2a) - keine solche Überlappung von zwei Konstruktionen vor, so dass zwar die Voranstellung der Objekt-PP-Fügung ohne Weiteres möglich ist, die Extraktion der PP aber weniger annehmbar erscheint. Die beiden Konstruktionen, die miteinander verschmelzen können, sind die beiden folgenden:

(16) $[\mathrm{NP}$ PP $] \mathrm{NP}$

z.B. eine Hausarbeit über Willy Brandt, ein Buch über Helmut Schmidt

(17) $[\mathrm{V}$ PP ] VP

z.B. über Willy Brandt schreiben, aber: *über Helmut Schmidt bestellen

Als Folge der Instanziierungsmöglichkeit zweier Muster, die sich die PP teilen, kann bei (10a) nicht nur eine Hausarbeit über Willy Brandt die Stellung im Satz wechseln kann, sondern auch die PP über Willy Brandt kann extrahiert sowie erfragt werden, wie (10c-d) belegen. In (10a) verschmelzen (16) und (17) also miteinander zu einer komplexen Konstruktion, die zwei Muster enthält. Die Verschmelzung in (10a) ist dadurch möglich, dass einerseits eine Hausarbeit über Willy Brandt Muster (16) instanziiert, andererseits über Willy Brandt schreiben, also schreiben in Kombination mit der idiomatisch verfestigten Präposition über, zugleich Satzmuster (17) instanziiert. In (11a) liegt dagegen nur eine Konstruktion, nämlich Muster (16), vor. Die Tatsache, dass (11c) ?? Über Helmut Schmidt möchte Doris ein Buch bestellen problematischer ist als (10c), erklärt sich demnach daraus, dass in diesem Satz keine zwei Muster miteinander verschmelzen, nur ein Buch über Helmut Schmidt (vgl. engl. a book about Nixon in (1)-(2)) ist eine sinnvolle Fügung, nicht aber *über Helmut Schmidt bestellen.

Fragesätze wie (10d) Über wen schreibt Paul seine Hausarbeit? müssen von Extraktionen unterschieden werden. Dass ein Satz wie Paul schreibt seine Hausarbeit über wen? unüblich ist, hat vielmehr umgekehrt mit der stark eingeschränkten Integrierbarkeit von Fragewörtern in NPs zu tun. Fragesätze stellen einen eigenständigen Konstruktionstyp dar, der 
keine Konstruktionsverschmelzung voraussetzt, weshalb sie auch anderen semantischen und informationsstrukturellen Einschränkungen unterliegen als Extraktionen, vgl. Über wen habt ihr einen Film gesehen? aber??/*̈̈ber wen habt ihr den Film gesehen? (Klein 1983, S. 47).

\section{4. $\quad$ Ein Dependenzmodell}

Die uns interessierende Wortstellungsvariation der PP müsste gemäß den obigen Überlegungen immer dann möglich sein, wenn die Bedingung für die Konstruktionsverschmelzung erfüllt ist, nämlich die Instanziierung von zwei Mustern, die strukturell in der PP überlappen. Die dem DEREKO entnommenen Beispielsätze (18)-(19) mit erzählen und lesen (vgl. eine Geschichte über etwas und über etwas erzählen/lesen) bestätigen das, sowohl die Voranstellung der Objekt-PP-Fügung als auch die Extraktion der PP erweisen sich als möglich und sind auch belegt:

(18a) Die darauffolgende Geschichte über die Notbremse im Zug und den Reiz des Verbotenen erzählte die Künstlerin mit äusserst überzeugendem Schauspiel und viel Humor. (St. Galler Tagblatt, 17.11.2008, S. 33)

(18b) Über ihre Anfänge hat mir ein befreundeter Kamer[a]mann eine schöne Geschichte erzählt. (die tageszeitung, 6.9.2001, S. 15)

(19a) Eine Geschichte über die Familie Krupp lesen Sie auch in unserem Magazin am kommenden Wochenende. (Nürnberger Nachrichten, 18.3.2009, S. 7)

(19b) Über Juan Reile stand vor kurzem auf dieser Seite eine Geschichte zu lesen. (Nürnberger Nachrichten, 3.4.2013, S. 34)

Dagegen verhalten sich wie (11a) Doris bestellt ein Buch über Helmut Schmidt, wo die Objekt-PP-Fügung ein Buch über Helmut Schmidt leichter vorangestellt und erfragt werden kann als die PP (s. (11c-d)), auch andere Sätze, die außer dem Muster (16) kein präpositionales Verbgefüge mit über realisieren, vgl. etwa (ein Buch über Helmut Schmidt) kaufen / empfangen / drucken / binden / liefern / zerstören / wegschmeißen / bearbeiten / interpretieren usw. Jedes Mal erscheint eine Extraktion der PP nicht gemäß der Norm, und ich habe dafür in DEREKo bisher keine Belege gefunden. Formal vergleichbare Beispielsätze mit vorangestellter PP weisen keine Extraktion auf und die semantische Funktion der PP ist dementsprechend eine andere, vgl. die beiden folgenden Sätze mit kaufen:

(20a) Über stationäre Automaten können Pendler Zeitkarten bargeldlos kaufen. (Frankfurter Rundschau, 23.4.1998, S. 12)

(20b) Über die Plattform können Mitglieder reduzierte Markenartikel kaufen. (Rhein-Zeitung, 8.2.2011, S. 25)

Die Extraktionsmöglichkeit in Sätzen mit Verben wie lesen bleibt auch dann bestehen, wenn zwei PPs aneinandergereiht werden, unter der Bedingung, dass sie vom selben nominalen Bezugswort abhängen (die Beispiele entnehme ich Pafel 1995, S. 147f.):

(21a) Ich habe alle Bücher von Lothar Gall über Bismarck gelesen.

(21b) Von Lothar Gall habe ich alle Bücher über Bismarck gelesen.

(21c) Über Bismarck habe ich alle Bücher von Lothar Gall gelesen. 
Sowohl die von-PP als auch die über-PP hängen von alle Bücher ab, über Bismarck ist kein Attribut zu Lothar Gall. ${ }^{8}$

Anhand einer Dependenzanalyse im Sinne von Lucien Tesnière (1966) kann der konstruktionelle Unterschied zwischen (10a) Paul schreibt eine Hausarbeit über Willy Brandt und (11a) Doris bestellt ein Buch über Helmut Schmidt wie folgt veranschaulicht werden:9

(22a) (Paul schreibt) [eine Hausarbeit [über Willy Brandt]].

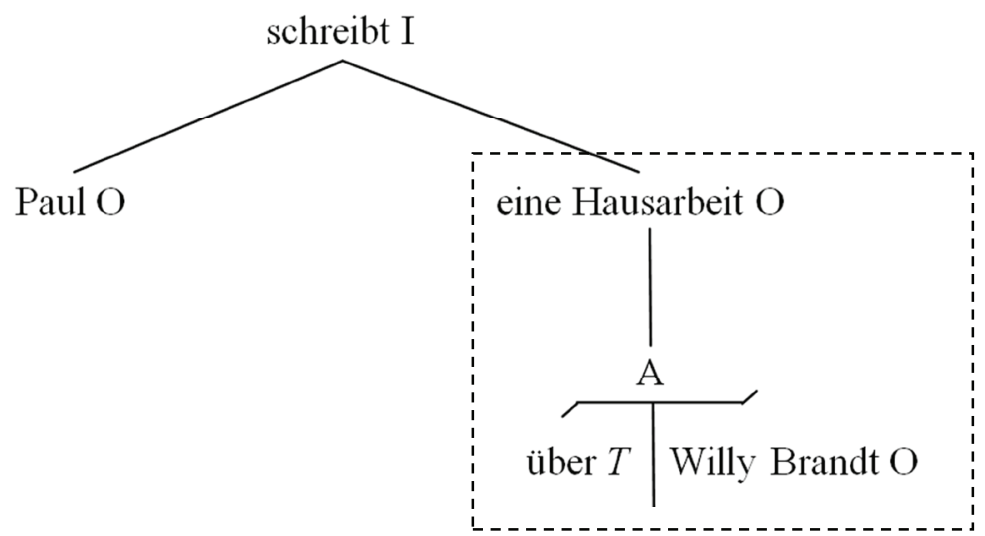

(22b) (Paul) schreibt über Willy Brandt.

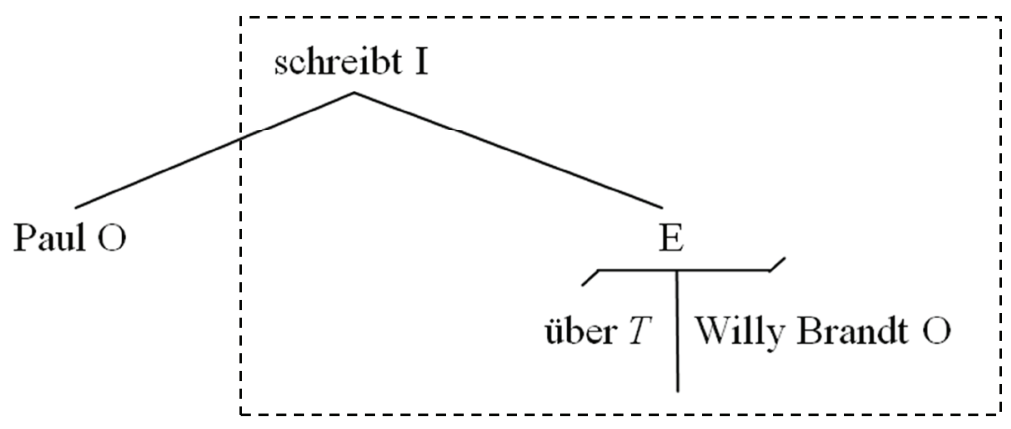

S Satz (21c) versieht Pafel (1995, S. 148) mit einem Fragezeichen. Der Satz erscheint mir aber genauso akezptabel wie Über Bismarck habe ich alle Bücher gelesen. Möglicherweise macht die von-PP als Teil des DO den Satz etwas schwerer verarbeitbar, in informationsstruktureller Hinsicht ist er aber mit Über Bismarack habe ich Lothar Galls Bücher gelesen vergleichbar. Nach Pafel (1995, S. 171) stellt (von) Lothar Gall übrigens ein direktes Argument“ von alle Bücher dar, über Bismarck hingegen ein „,indirektes Argument“.

9 Man kann den Unterschied auf unterschiedliche Art und Weise darstellen. Ich wähle Tesnières Analysemodell hier zu rein illustrativen Zwecken (vgl. Weber 1992), weil es relativ theoriefrei ist und kaum einen das Verständnis erschwerenden Formalismus voraussetzt. Das Modell stellt das Verb bei der syntaktischen Analyse in den Mittelpunkt des Satzes. Die Bedeutung der Buchstaben ist: $\mathrm{I}=$ Verb, $\mathrm{O}=$ Substantiv, A = Adjektiv, $T=$ Translativ (s. Tesnière 1966, $\S 33$ und $\S \S 151-157$ zur Translation). Mittels eines Translativs (z.B. einer Präposition) wird eine Wortart in eine andere Wortart überführt, z.B. Hamburg $(\mathrm{O})>$ in Hamburg $>$ (E) in Das gab es noch nie in Hamburg oder Jürgen Habermas $(\mathrm{O})>$ von Jürgen Habermas (A) in Da kommt ein Buch von Jürgen Habermas gerade recht. In den Strukturbäumen veranschaulicht das eingezeichnete $T$ jeweils die Translation. 
(22c) (Paul) schreibt eine Hausarbeit über Willy Brandt.

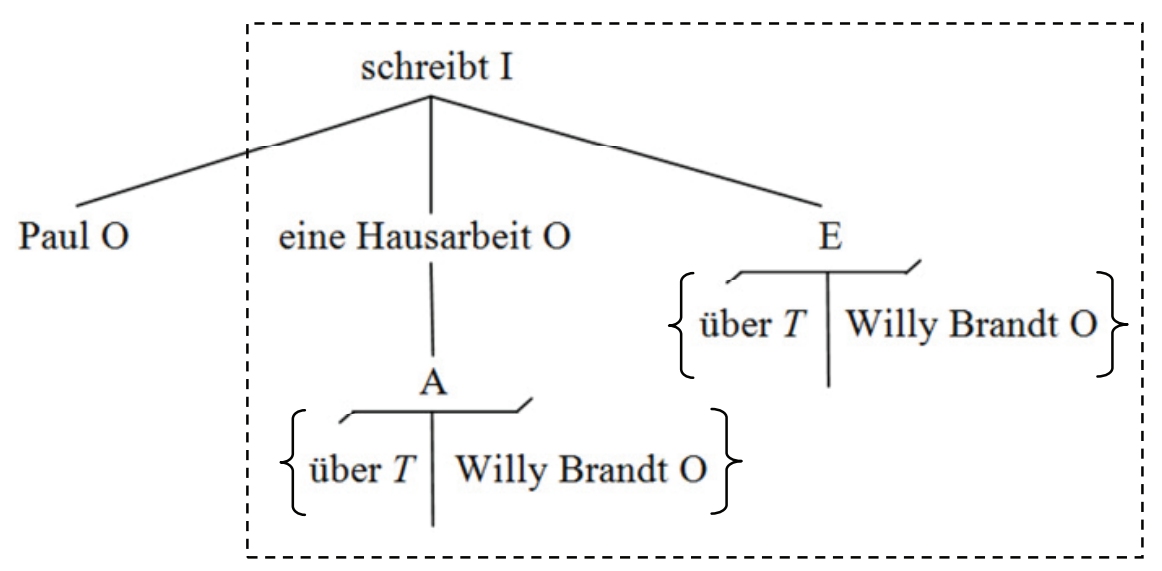

(23) Doris bestellt [ein Buch [über Helmut Schmidt]].

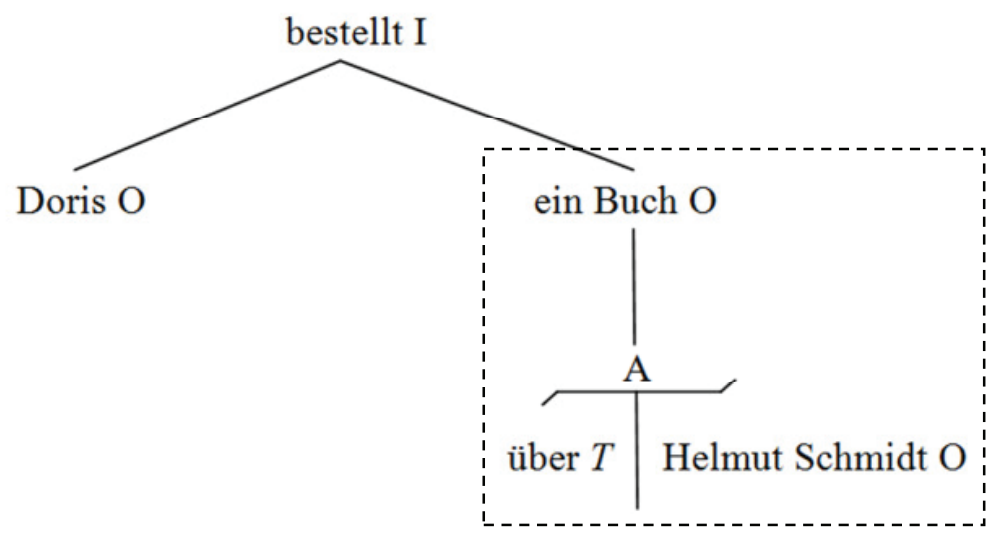

Die PP über Willy Brandt in (22c) erfüllt also sowohl die Funktion eines Attributs (A) zur NP eine Hausarbeit als auch die Funktion einer Adverbialphrase (E) beim Verb schreibt. In Satz (23) mit dem Verb bestellen kann die PP über Helmut Schmidt dagegen nur ein Attribut (A) zur NP ein Buch sein.

Anhand von Strukturbäumen (,Stemmata“) wie (22)-(23) lässt sich eine generelle Hypothese über Konstruktionen und Wortstellung aufstellen. Es handelt sich um eine Annahme über das implizite ,idiomatische Wissen“ (Coseriu 1985, S. xxviii-xxx), das Sprecher über die Konstruktionen besitzen, die in einem Satz instanziiert werden: In ein und demselben Satz können zwei Konstruktionen dergestalt miteinander verschmelzen, dass der Satz konstruktionell ,geschichtet" bleibt und eine entsprechende Wortstellungsvariation aufweist. Die Bedingung für die Konstruktionsverschmelzung besteht darin, dass die beiden Muster ein strukturelles Element gemeinsam haben (im uns hier interessierenden Fall eine PP). Insofern stellt das Ergebnis der Verschmelzung einen speziellen Typ von ApoKoinu-Konstruktion dar. ${ }^{10}$

10 Eine Erscheinung, die sich auch im heutigen Deutsch an mancher Stelle noch findet, vgl. Duden (2006, § 2016) zu mündlichen Konstruktionen vom Typ des is was furchtbares is des. Dabei wird ein zwei Konstruktionen gemeinsames Element (das „Koinon“) nur einmal realisiert. Im erwähnten Beispiel aus 


\section{Graduelle Unterschiede}

In Abschnitt 2 wurde darauf hingewiesen, dass es bei der Beurteilung der Wohlgeformtheit und Semantizität von Sätzen Stufen der Akzeptabilität gibt, die sich kontextuell manipulieren lassen. Der oben skizzierte Erklärungsansatz berücksichtigt solche graduellen Unterschiede. Konstruieren wir dazu die folgende Reihe von Sätzen mit einer Objekt-PPFügung mit der Präposition von:

(24a) Er hat das Fahrrad von Karl geerbt.

(24b) Er hat das Fahrrad von Karl gekauft.

(24c) Er hat das Fahrrad von Karl gesehen.

(24d) Er hat das Fahrrad von Karl zerstört.

Es hat den Anschein, dass die Extraktionsmöglichkeit der PP von (24a) zu (24d) allmählich abnimmt. Dem entspricht die Feststellung, dass bei identischer Realisierung der Konstruktion (16) [NP PP]NP (das Fahrrad von Karl) die Instanziierungsmöglichkeit der Konstruktion (17) [V PP]VP abnimmt. Tatsächlich sind von jmdm. erben und von jmdm. kaufen relativ verfestigte präpositionale Verbgefüge des Deutschen, was aber von von jmdm. sehen nicht in gleichem Maße und von von jmdm. zerstören kaum gesagt werden kann. Entsprechend ist die Interpretation von das Fahrrad von Karl im Sinne eines Besitzverhältnisses in (24a) und (24b) weniger zwingend als in (24c) und (24d). Vgl. die unterschiedlichen Wortfolgen in den folgenden Belegen mit erben und kaufen:

(25a) sein Mieter erhalte unberechtigt Sozialhilfe - dieser habe gerade viel Geld von seiner angeblich verstorbenen Mutter geerbt. (Hamburger Morgenpost, 1.9.2011, S. 03)

(25b) Von ihrem Vater hat sie 180 Kühe geerbt. (St. Galler Tagblatt, 3.8.2011, S. 8)

(25c) Von seinem Vorgänger George W. Bush hat Obama ein gigantisches Defizit geerbt. (Süddeutsche Zeitung, 23.2.2009, S. 17)

(26a) Ja, jeder könnte unbesehen ein gebrauchtes Auto von ihm kaufen. (Süddeutsche Zeitung, 24.8.2000, S. 13)

(26b) „Von jemandem wie ihm würde ich nicht einmal ein gebrauchtes Auto kaufen.“(Nürnberger Zeitung, 8.12.2009, S. 23)

(26c) Von Nordkorea will Gadafi sowjetische SCUD-Raketen kaufen. (Neue Kronen-Zeitung, 27.2.1996, S. 3)

(25b) und (26b) können als Beispiele von Extraktion analysiert werden (vgl. 180 Kühe von ihrem Vater, ein gebrauchtes Auto von jemandem wie ihm), aber eine solche Analyse ist nicht zwingend. (25c) und (26c) sind dagegen kaum noch als Beispiele von Extraktion analysierbar.

\section{Schlussbetrachtungen}

Einige weitere Punkte sollen abschließend noch hervorgehoben werden. Erstens gibt es natürlich auch andere Gründe für die Stellungsvariation von PPs, und auch für Extraktion. Konstruktionsverschmelzung liegt nur dann vor, wenn zwei sich überlappende konstruk-

dem Duden kann das gemeinsame Element ohne Weiteres den beiden linear verschränkten Sätzen zugeordnet werden; in den hier besprochenen Fällen von Konstruktionsverschmelzung nimmt die gemeinsame PP darüber hinaus zwei unterschiedliche hierarchische Positionen ein. 
tionelle Muster gleichwertig im Satz vertreten sind. Unter (27) z.B. ist ersichtlich, dass die PP über die Heizung vorangestellt und erfragt werden kann:

(27a) Hans hängt das Handtuch über die Heizung.

(27b) *Das Handtuch über die Heizung hängt Hans.

(27c) Über die Heizung hängt er das Handtuch.

(27d) Worüber (über was) hängt Hans das Handtuch?

(27e) *Über die Heizung möchte Hans hängen.

(28f) *Hans hängt.

(28g) Was hängt Hans über die Heizung?

Die Erklärung für (27a-g) bereitet keine Schwierigkeiten. In (27c) Über die Heizung hängt er das Handtuch liegt keine Extraktion vor, in (27a) wird nur die Konstruktion (17) [V PP]VP instanziiert, nicht aber Konstruktion (16) [NP PP]NP. Die PP über die Heizung ist Teil des Verbalnukleus [[über X] hängen], der die transitive Bindungsfähigkeit und damit die Anbindung des DO das Handtuch allererst ermöglicht (vgl. * Er hängt das Handtuch). Entsprechend kann nur die PP über die Heizung, nicht aber das DO und die PP zusammen (das Handtuch über die Heizung) an den Anfang des Satzes gestellt werden (vgl. dagegen Das Handtuch über der Heizung gehört mir, wobei der Dativ ein weiteres Indiz dafür ist, dass in etwas über die Heizung hängen keine Überlappung einer PP in zwei Konstruktionen vorliegt). Damit unterscheidet sich (27a) grundsätzlich von beispielweise (10a) Paul schreibt eine Hausarbeit über Willy Brandt, da die Anbindung des DO eine Hausarbeit in diesem Satz auf keinen komplexen Verbalnukleus [[über X] schreiben] angewiesen ist. Wieder anders verhält es sich mit (28) und (29), denen offensichtlich keine der beiden Konstruktionen (16) und (17) zugrunde liegen:

(28) Kenner essen den Fisch mit den Fingern - und verspeisen ihn mit Haut und Flossen. (Hamburger Morgenpost, 6.3.2008, S. 27)

(29) Die Beamten brachen die Handschellen mit Werkzeug auf. (Hamburger Morgenpost, 7.4.2010, S. 11)

Möglich sind die Voranstellung der PP (Mit den Fingern essen sie den Fisch; Mit Werkzeug brachen sie die Handschellen auf) und des DO (Den Fisch essen Kenner mit den Fingern; Die Handschellen brachen die Beamten mit Werkzeug auf). DO und PP können aber nicht zusammen vorangestellt werden: *Den Fisch mit den Fingern essen Kenner; *Die Handschellen mit Werkzeug brachen die Beamten auf. Entsprechend kann die Voranstellung der PP keine Extraktion sein. Ohnehin scheinen PPs, die ein Instrument bezeichnen, ihre Stellung im Satz in der Regel nicht aufgrund von Extraktion aus einer umfassenderen NP zu wechseln.

Zweitens kann die Extraktionsmöglichkeit aus unterschiedlichen, insbesondere semantischen Gründen eingeschränkt oder ggf. sogar blockiert sein, auch wenn Konstruktionsverschmelzung vorliegt, was ebenfalls auf die Gradualität des Phänomens hinweist. Das ist z.B. der Fall bei PPs mit der Präposition an. Als Variante für das indirekte Objekt im Dativ sind PPs mit an nicht ohne Weiteres extrahierbar. Satz (30) hat zwar zwei Lesarten - entweder hat die Verlobte die Briefe an eine Boulevardzeitung verkauft oder sie hat die Briefe, die an eine Boulevardzeitung gerichtet waren, verkauft: 
(30) Im April war eine frühere Verlobte Hewitts verhaftet worden, weil sie die Briefe an eine Boulevardzeitung verkaufen wollte. (Frankfurter Rundschau, 10.12.1998, S. 38)

Wird aber die PP extrahiert, ist nur noch die erste Lesart möglich, die zweite ist ausgeschlossen: weil sie an eine Boulevardzeitung die Briefe verkaufen wollte.

In den beiden folgenden Sätzen gibt es jeweils zwei PPs, die aufeinander folgen:

(31) Claudia Pechstein schreibt an einem Buch über ihr Leben. (Nürnberger Nachrichten, 19.1.2010, S. 23)

(32) Das kleine Mädchen Emma liest in einem Buch über diese Geschichte und ist wild entschlossen, diesen armen Tieren zu helfen. (Rhein-Zeitung, 25.11.2009)

Die mögliche Wortstellungsvariation ist in beiden Sätzen anders: In (31) kann nur die Fügung an einem Buch über ihr Leben insgesamt an den Satzanfang rücken, nicht aber die PP über ihr Leben allein (dagegen wäre der transitive Satz Über ihr Leben schreibt sie ein Buch wohlgeformt). In (32) kann außer der Fügung in einem Buch über diese Geschichte auch die PP über diese Geschichte linksversetzt werden. Während also die Extraktion in (32) möglich ist, erscheint sie in (31) blockiert. Der Grund ist wiederum semantisch: Wenn ausgedrückt wird, dass jemand an einem Buch über sein Leben schreibt, dann kann das nur heißen, dass das Buch vom Leben dieser Person handelt. Wenn dagegen ausgedrückt wird, dass jemand in einem Buch über irgendeine Geschichte liest, dann kann das ein Buch nur über diese Geschichte sein, aber möglich ist auch, dass das Buch von etwas anderem handelt und die Geschichte darin z.B. kurz Erwähnung findet. Tatsächlich erzwingt die Linksversetzung der über-PP in (32) - vgl. Über diese Geschichte liest sie in einem Buch - so gut wie ausschließlich letztere Interpretation.

Drittens bietet der hier vorgeschlagene Ansatz eine mögliche Erklärung für die Tatsache, dass tiefer ,eingebettete“ PPs nicht extrahierbar sein können, vgl.:

(33a) Der Schüler schreibt eine Besprechung über ein Buch über Nixon.

(33b) Über ein Buch über Nixon schreibt der Schüler eine Besprechung.

(33c) *Über Nixon schreibt der Schüler eine Besprechung über ein Buch.

Der Grund dafür, dass die PP über Nixon nicht extrahierbar ist, ist nicht so sehr, dass die PP tiefer eingebettet ist als die PP über ein Buch [über Nixon], sondern dass die Extraktion von über Nixon keinen Sinn ergibt, im Gegensatz zur Extraktion der PP über ein Buch über Nixon, die sinnvoll ist. Die Verschmelzung der Muster (16) und (17) in (33a), die auch (33b) kennzeichnet, folgt dem grundlegenden und allgemeinen Prinzip sprachlicher Zeichen, das besagt, dass einer Form eine Bedeutung entspricht - und damit sind nicht nur Morpheme und Wörter, sondern auch Wortgruppen und Sätze gemeint.

Viertens ist die Instanziierung von Konstruktionen, die miteinander verschmelzen können, insofern variabel, als deren verbale Leerstelle nicht ausschließlich durch bestimmte Lexeme besetzt wird. Zwar stellen Sätze mit lesen (über) und schreiben (über) sowie ihren Entsprechungen in anderen Sprachen die Standardbeispiele in der einschlägigen Forschungsliteratur zur Extraktion dar. Die semantische Funktion dieser Verben scheint aber wenigstens teilweise auf Verben mit verwandter Bedeutung vererbbar zu sein. So gilt dieselbe Wortstellungsvariation, die einen Satz wie Paul schreibt eine Hausarbeit über Willy Brandt kennzeichnet, wenn der Satz mit archisememischen Verben wie machen oder anfertigen („Hyperonymen“) oder umgekehrt mit „Hyponymen“ wie abfassen und verfas- 
sen gebildet wird, auch wenn die entsprechenden präpositionalen Verbgefüge nicht - oder nicht in gleichem Maße - lexikalisiert sind.

Schließlich erscheint es sinnvoll, zu untersuchen, ob Konstruktionsverschmelzung mit der ihr eigenen Wortstellungsvariation auf verschiedenen Ebenen grammatischer Strukturierung vorkommt. Es dürfte z.B. kein Zufall sein, dass auch im Bereich der Wortbildung eine ähnliche Fusion zweier Konstruktionen festgestellt werden kann (ergänzend zu u.a. Booij 2010, S. 41-50). Beispielsweise können im Deutschen die beiden Kompositionsmuster unter (34) und (35) miteinander verschmelzen:

(34) $[$ [NDeterminans $]+[$ NDeterminatum $]]$

z.B. Wettervorhersage, Tagestemperatur, Blutdruck, Fachhandel

(35) $[$ [ADeterminans $]+[$ NDeterminatum $]]$

z.B. Kurzvorhersage, Höchsttemperatur, Niedrigdruck, Großhandel

Das Ergebnis sind Trikomposita (s. Willems 1990, S. 55-57), die anscheinend ein größeres $\mathrm{Ma} ß$ an Wortstellungsvariation als monokonstruktionelle Wortbildungskonstruktionen aufweisen, wobei bestimmte semantische oder pragmatische Unterschiede zwischen den beiden Varianten gemäß dem zu Anfang des Beitrags erwähnten „Dominanz“-Prinzip allerdings nicht ausgeschlossen sind; vgl. die folgenden in Texten und auf deutschen Internetseiten belegten Beispiele:

(36) Wetterkurzvorhersage $\approx$ Kurzwettervorhersage

Tageshöchsttemperatur $\approx$ Höchsttagestemperatur

Blutniedrigdruck $\approx$ Niedrigblutdruck

Bluthochdruck $\approx$ Hochblutdruck

Teilnehmerhöchstzahl $\approx$ Höchstteilnehmerzahl

Fleischgroßmarkt $\approx$ Großfleischmarkt

Milchfrischprodukt $\approx$ Frischmilchprodukt

Personenkleinwagen $\approx$ Kleinpersonenwagen

Fachgroßhandel $\approx$ Großfachhandel ${ }^{11}$

Die Hypothese über eine generelle Korrelation zwischen Konstruktionsverschmelzung und Wortstellungsvariation im Deutschen stelle ich hier in den Raum, weitere Untersuchungen sind erforderlich, um darüber empirisch breit fundierte Aussagen machen zu können.

\section{Literatur}

Altmann, Hans (1981): Formen der „Herausstellung“ im Deutschen: Rechtsversetzung, Linksversetzung, freies Thema und verwandte Konstruktionen. Tübingen: Niemeyer.

Booij, Geert (2010): Construction Morphology. Oxford: Oxford University Press.

Bouchard, Denis (1995): The semantics of syntax. Chicago: The University of Chicago Press.

Bouchard, Denis (2013): The nature and origin of language. Oxford: Oxford University Press.

Broekhuis, Hans (2005): Extraction from subjects: Some remarks on Chomsky's 'On phases'. In: Broekhuis, Hans et al. (Hg.): Organizing Grammar. Linguistic Studies in Honor of Henk van Riemsdijk. Berlin/New York: Mouton de Gruyter. S. 59-68.

11 Dagegen nur Billiglederwaren (*Lederbilligwaren); semantisch bedingte Blockierungen sind ebenfalls zu berücksichtigen (z.B. Altstadtteil, *Stadtaltteil). Bei noch komplexeren Dekomposita scheint die Wortstellungsvariation eingeschränkt zu sein, vgl.: Leichtkraftfahrzeug (*Kraftleichtfahrzeug), Kleinkraftfahrzeug (??/*Kraftkleinfahrzeug) usw. 


\section{Klaas Willems}

Chomsky, Noam (1986): Barriers. Cambridge, MA: MIT Press.

Chomsky, Noam (2005): On phases. Manuskript. MIT.

Coene, Ann/Willems, Klaas (2006): Konstruktionelle Bedeutungen. Kritische Anmerkungen zu Adele Goldbergs konstruktionsgrammatischer Bedeutungstheorie. In: Sprachtheorie und germanistische Linguistik 16 (1), S. 1-35.

Coseriu, Eugenio (1985): Linguistic competence: what is it really? In: The Modern Language Review 80 (4), S. xxv-xxxv.

Dabrowska, Ewa (2010): Naïve vs. expert intuitions: An empirical study of acceptability judgments. In: The Linguistic Review 27, S. 1-23.

Erteschik-Shir, Nomi (1981): More on extractability from Quasi-NPs. In: Linguistic Inquiry 12, S. 665-670.

Goldberg, Adele (2003): Constructions: a new theoretical approach to language. In: Trends in Cognitive Sciences 7 (5), S. 219-224.

Klein, Maarten (1983): Vooropstaande PP's en thematische relaties. In: Gramma 7 (1), S. 41-50.

Müller, Stefan (2003): Mehrfache Vorfeldbesetzung. In: Deutsche Sprache 31, S. 29-62.

Pafel, Jürgen (1995): Kinds of extraction from Noun Phrases. In: Lutz, Uli/Pafel, Jürgen (Hg.): On extraction and extraposition in German. Amsterdam: Benjamins. S. 145-177.

Pustejovsky, James (1995): The Generative Lexicon. Cambridge, MA/London: MIT Press.

Tesnière, Lucien (1966): Éléments de syntaxe structurale. Préface de Jean Fourquet. 2. Aufl. Paris: Klincksieck.

Van de Velde, Freek (2012): PP extraction and extraposition in Functional Discourse Grammar. In: Language Sciences 34 (4), S. 433-454.

Wasow, Thomas/Arnold, Jennifer (2005): Intuitions in linguistic argumentation. In: Lingua 115, S. 1481-1496.

Weber, Heinz J. (1992): Dependenzgrammatik. Ein Arbeitsbuch. Tübingen: Narr.

Welke, Klaus (1988): Einführung in die Valenz- und Kasustheorie. Leipzig: VEB Bibliographisches Institut.

Willems, Klaas (1990): Tageshöchsttemperaturen, Billigst-Flüge und Halbknaben. Zur Syntax, Semantik und Stilistik eines beliebten Wortbildungsmodells im heutigen Deutsch. In: Deutsche Sprache 18, S. 52-75.

Willems, Klaas (2011): Meaning and interpretation: The semiotic similarities and differences between Cognitive Grammar and European Structural Linguistics. In: Semiotica 185, S. 1-50.

Willems, Klaas/Coene, Ann (2006): Satzmuster und die Konstruktionalität der Verbbedeutung. Überlegungen zum Verhältnis von Konstruktionsgrammatik und Valenztheorie. In: Sprachwissenschaft 31 (3), S. 237-272.

Ziem, Alexander/Lasch, Alexander (2013): Konstruktionsgrammatik. Konzepte und Grundlagen gebrauchsbasierter Ansätze. Berlin/New York: de Gruyter.

Prof. Dr. Klaas Willems

Universiteit Gent

Fachbereich Sprachwissenschaft Allgemeine Sprachwissenschaft

Blandijnberg 2

B-9000 Gent

E-Mail: Klaas.Willems@UGent.be 